\title{
Teratoma Removal, Steroid, IVIG, Rituximab and Tocilizumab (T-SIRT) in Anti-NMDAR Encephalitis
}

\author{
Woo-Jin Lee $^{1} \cdot$ Soon-Tae Lee ${ }^{1} \cdot$ Yong-Won Shin ${ }^{1,2,3} \cdot$ Han Sang Lee ${ }^{1} \cdot$ Hye-Rim Shin ${ }^{4} \cdot$ Do-Yong Kim ${ }^{1,5} \cdot$ Soyun Kim ${ }^{1,5}$. \\ Jung-Ah Lim ${ }^{6} \cdot$ Jangsup Moon ${ }^{1,5} \cdot$ Kyung-II Park $^{1,5,7} \cdot$ Hee Seung Kim ${ }^{8} \cdot K_{\text {Kon Chu }}^{1,5}$ (10 $\cdot$ Sang Kun Lee ${ }^{1}$
}

Published online: 3 September 2020

(C) The American Society for Experimental NeuroTherapeutics, Inc. 2020

\begin{abstract}
In anti-N-methyl-D-aspartate receptor (NMDAR) encephalitis, we analysed the efficacy of a combined immunotherapy protocol consisting of teratoma removal, steroid, intravenous immunoglobulin (IVIG), rituximab and tocilizumab (TSIRT). This cohort study included seventy-eight consecutive patients treated for anti-NMDAR encephalitis between Jan 2014 and Oct 2019 in a national referral hospital. Detailed 2-year disease time course was analysed using Clinical Assessment Scale for Autoimmune Encephalitis (CASE) scores at every 2 weeks for 12 weeks from baseline, every month for the next 3 months and then every 3 months. Treatment regimens at each time point were categorized as SI, SIR, or SIRT with/without teratoma removal (T). Adverse events were classified according to the Common Terminology Criteria for Adverse-Events (CTCAE v5.0), where a severe adverse event was defined as an adverse event with CATAE grade 4. In a linear mixed model analysis, using the SIRT regimen was more effective than SIR or SI regimens in lowering CASE scores $(P<0.001$ and $P=0.001$, respectively). The presence of teratoma $(P=0.001)$, refractory status epilepticus $(P<0.001)$ and a higher CASE score at baseline $(P<0.001)$ predicted a higher CASE score at each time point. Completion of the (T)-SIRT regimen within 1 month of onset resulted in better 1-year improvements in CASE score $(P<0.001)$ and modified Rankin scale scores $(P=0.001)$, compared to those of using other regimens within 1 month or delaying teratoma removal for more than 1 month. Pneumonia was a frequent adverse event $(52 / 78$, $66.7 \%$ ) in the whole study population and neutropenia was frequent during SIRT $(11 / 52,21.2 \%)$, but the regimen was well tolerated in most patients. We concluded that the early application of combined immunotherapy consisting of TSIRT had better efficacy than was found for delayed or partial application of this combination in anti-NMDAR encephalitis.
\end{abstract}

Key Words Anti-N-methyl-D-aspartate receptor $\cdot$ autoimmune encephalitis $\cdot$ immunotherapy $\cdot$ prognosis $\cdot$ ovarian teratoma.

Woo-Jin Lee and Soon-Tae Lee share the first authorship.

Electronic supplementary material The online version of this article (https://doi.org/10.1007/s13311-020-00921-7) contains supplementary material, which is available to authorized users.

Kon Chu

stemcell.snu@gmail.com

Sang Kun Lee

sangkun2923@gmail.com

1 Department of Neurology, Seoul National University Hospital, 101, Daehak-ro, Jongno-gu, Seoul 110-744, South Korea

2 Center for Hospital Medicine, Seoul National University Hospital, Seoul, South Korea

3 Department of Neurosurgery, Seoul National University Hospital, Seoul, South Korea

4 Department of Neurology, Dankook University Hospital, Cheonan, South Korea

5 Laboratory for Neurotherapeutics, Center for Medical Innovations, Biomedical Research Institute, Seoul National University Hospital, Seoul, South Korea

6 Department of Neurology, Cham Joeun Hospital, Gwangju, South Korea

7 Department of Neurology, Seoul National University Hospital Healthcare System Gangnam Center, Seoul, South Korea

8 Department of Obstetrics and Gynecology, Seoul National University Hospital, Seoul, South Korea 


\section{Introduction}

Anti-N-methyl-D-aspartate receptor (anti-NMDAR) encephalitis is the most common and serious type of autoimmune encephalitis (AE) and is associated with ovarian teratoma in approximately $40 \%$ of the cases [1-5]. Recent advances are establishing immunotherapy regimens, such as the first-line (steroid, intravenous immunoglobulin (IVIG) and plasma exchange), second-line (rituximab and cyclophosphamide) and next-line treatments (tocilizumab and bortezomib) [3, 6, 7]. In particular, tocilizumab, a monoclonal antibody that blocks the interleukin- 6 receptor, is a novel line of treatment used in patients with insufficient responsiveness to first-line treatment and rituximab [8] and has been shown to be promisingly effective in new-onset refractory status epilepticus [9]. Because 19.4-25.5\% of anti-NMDAR encephalitis patients are still refractory to the current immunotherapies $[3,10,11]$ and a significant portion of patients results in prolonged neurological deficits such as executive dysfunction, memory impairment, behavioural problems and sleep disturbances [12], more optimized and effective sequences of immunotherapy are necessary.

To develop a more effective regimen, some critical issues should be resolved. First, although we know that combined immunotherapy is better in refractory cases, we need to know how fast we should combine a next-line immunotherapy with the previous one $[3,7,8]$. Second, because regimens at different levels are used for short intervals, outcomes reflect a delayed effect of the previous regiment, and thus the composite effect of combined immunotherapies should be analysed. Third, with the introduction of next-line novel immunotherapies, such as tocilizumab, treatments should be integrated into a conventional treatment protocol if this improves outcomes. Fourth, because previous outcome analyses used the modified Rankin scale (mRS), which is largely dependent on motor functions and is not completely suitable for assessing $\mathrm{AE}[3,6-8,10]$, the Clinical Assessment Scale in Autoimmune Encephalitis (CASE) [11], a novel severity score for AE that represents a comprehensive scoring system with a score range of $0-27$, could be an alternative tool for more precise outcome analysis. Finally, non-immunotherapeutic modifiers, such as the removal of the teratoma and the presence of refractory status epilepticus, should be considered when analysing the clinical outcomes of anti-NMDAR encephalitis because their effects have not been elucidated $[4,9,13,14]$.

To address these questions, we performed a prospective cohort analysis of anti-NMDAR encephalitis. Here, we analyse the treatment outcomes and their modifiers, taking account the time sequences of each immunotherapy, the use of novel biologics, new outcome analysis tools and supportive care. We demonstrate the optimal treatment regimen and identify factors that have a significant impact on the clinical course of anti-NMDAR encephalitis.

\section{Materials and Methods}

\section{Study Population}

This study included all consecutive patients who were in the prospective autoimmune encephalitis cohort of Seoul National University Hospital (SNUH) and treated for definite anti-NMDAR encephalitis in SNUH between January 1, 2014, and October 31, 2019. Each clinical diagnosis of definite anti-NMDAR encephalitis was re-confirmed according to recent criteria $[15,16]$. The presence of antiNMDAR autoantibodies in patients' serum and CSF was detected using immunohistochemistry staining of rat brain sections and a cell-based immunocytochemistry assay kit (Euroimmune Ag, Germany), as described in our previous studies $[7,8,11,16-19]$. This study was approved by the institutional review board of SNUH, and written informed consent was obtained from all enrolled patients or their legal surrogate.

\section{Analysis of Clinical and Laboratory Profiles}

To evaluate changes in clinical severity over time, CASE and mRS scores were obtained at each time point designated as follows: at the time of initiation of immunotherapy (baseline), every 2 weeks (window time of \pm 2 days) for 3 months, every month (window time of \pm 5 days) for the next 3 months and then every 3 months (window time of \pm 2 weeks) for the remaining period. CASE is a novel severity scale for AE score consisting of nine items (seizure, memory dysfunction, psychiatric symptoms, consciousness, language problems, dyskinesia/dystonia, gait instability and ataxia, brainstem dysfunction and weakness). Score range for each item is 0-3 making the total score range of $0-27$. The reproducibility of medical record-based CASE scoring was established in a previous study [11]. Two neurologist who were experts in autoimmune encephalitis (W.J.L. and S.T.L. or K.C.) independently performed the scoring retrospectively based on patients' medical records, and consensus was achieved after discussion in any discrepant cases. Missing values were left blank.

Laboratory data including baseline MRI and CSF profiles were reviewed. CSF analysis included CSF whiteblood cell (WBC) levels, protein levels and the presence of any abnormality in CSF (WBC count of $\geq 5$ cells $/ \mathrm{mm}^{3}$ or protein level of $\geq 40 \mathrm{mg} / \mathrm{dL}$ ) or CSF WBC count of $>$ 20 cells $/ \mathrm{mm}^{3}[3,7,8,10,15]$. Abnormality in MRI was defined as hyperintensities in medial temporal lobes or in multifocal areas involving the neocortex on T2-weighted fluid-attenuated inversion recovery sequences $[3,7,8,10$, 15]. Development of refractory status epilepticus (refractory status epilepticus) defined as prolonged SE refractory to appropriate doses of $\geq 2$ types of antiepileptic drugs, or the use of ventilator, was also reviewed $[9,13]$. 


\section{Analysis of Treatment Profiles}

The protocol used to treat anti-NMDAR encephalitis was as follows. First, first-line immunotherapy agents, including corticosteroid and IVIG with or without plasma exchange, were administered [3, 6-8]. Second, the presence of a concomitant tumour, especially ovarian teratoma, was evaluated using pelvis computed tomography $(\mathrm{CT})$ or magnetic resonance imaging (MRI) evaluations, and the tumour was promptly removed when possible $[3,20]$. Third, rituximab and tocilizumab were administered as the second- and next-line immunotherapy regimens, respectively $[3,6-8]$.

The dosage of corticosteroid was a $1000 \mathrm{mg}$ of intravenous methylprednisolone for 3-5 days with or without maintenance of oral prednisolone. IVIG was administered at $1-2 \mathrm{~g} / \mathrm{kg}$ during 2-5 days per cycle. The timing of the next immunotherapy as well as the cycles and dosages of immunotherapy regimens was decided by expert physicians based on the patients' disease severity and responsiveness, the safety of each regimen and any sequelae that would develop in the absence of further clinical improvements. Rituximab was administered at $375 \mathrm{mg} / \mathrm{m}^{2}$ weekly for 4 cycles with or without additional monthly maintenance $[3,6-8,21]$. The standard dosage of tocilizumab was $8 \mathrm{mg} / \mathrm{kg}$ monthly and was started as reduced (4-6 mg/kg monthly) or split doses $(2-4 \mathrm{mg} / \mathrm{kg}$ repeated), especially in patients susceptible to hematologic or infectious adverse events (e.g. low absolute neutrophil [ANC] count [< $1500 / \mathrm{uL}]$, a previous history of decrement in ANC after receiving immunotherapy agent, or a recent history of a pneumonia or a urinary tract infection) $[8,21]$.

To screen for underlying tumours, the physician chose from among CT (chest, abdomen and pelvis), pelvis MRI, pelvis sonography (female) or testis sonography (male) $[7,8$, 21]. For female patients with insufficient responsiveness to sequential immunotherapies, pelvis MRI (or CT) was repeated to check for the delayed identification of teratoma.

The disease onset was operationally defined as the time point when the patient's clinical symptoms (memory deficits, altered mental status, or psychiatric symptoms) and laboratory findings (CSF WBC count of $\geq 5$ cells $/ \mathrm{mm}^{3}$, development of seizures, or abnormality in brain MRI) first met the diagnostic criteria for possible autoimmune encephalitis [15]. The time lag from symptom onset to the removal of a teratoma, the initiation of first-line immunotherapy or rituximab or tocilizumab and the number of cycles of each treatment regimen were reviewed $[7,8]$. The treatment regimen applied at each time point was classified as (T)-SI, (T)-SIR or (T)-SIRT, which indicates the evaluation and removal of teratoma ( $\mathrm{T}$, if present), the administration of steroid and IVIG (SI), rituximab (R) and tocilizumab (T), respectively. The presence of a teratoma at each time point was also reviewed. Delayed teratoma removal was defined as a 1-month or longer time lag from symptom onset to teratoma removal [10].

\section{Analysis of Clinical Outcomes}

Clinical outcomes at the last follow-up were classified according to CASE scores as excellent ( $0-4)$, moderate (5-9) or poor (10-27) or by mRS scores as favourable (0-2) or poor (3-6) $[3,6-8,10]$. Adverse events were classified and evaluated according to the Common Terminology Criteria for Adverse-Events (CTCAE v5.0). For instance, pneumonia was defined as an infectious disorder involving the lungs identified by clinical symptoms, increased opacity in chest $\mathrm{x}$-ray and elevated inflammation markers such as peripheral leukocyte count and C-reactive protein. Serious adverse events were defined as adverse events with CATAE grade $\geq 4$ [22]. Latent class linear modelling (LCLM) was applied to classify the patients into three groups according to changes in CASE score patterns over time [23]. Linear mixed model (LMM) analyses were performed to evaluate the effect of clinical factors, such as the treatment regimen or the presence of teratoma, on the CASE score changes over time. Changes in the CASE and the mRS scores between the baseline and 6 months (6-month $\triangle$ CASE and $\Delta$ mRS scores) and baseline and 1 year (1-year $\Delta$ CASE and $\Delta$ mRS scores) were also measured.

\section{Statistical Analysis}

The results are presented as the means \pm standard deviation, median (interquartile range, IQR) or number (percentage). All statistical analysis was performed by W.J.L. blinded to the patient information during the statistical analysis. As the correlation between CASE score changes and time was not linear nor logarithmic, square of time variable was introduced to build a quadratic function of CASE scores for time in the LCLM analysis. This model had better fitness than linear or logarithmic models, according to the Bayesian information criterion (BIC) values. Comparisons among the groups returned from LCLM were analysed using analysis of variance (ANOVA) followed by post hoc analysis using the least significant difference (LSD) or Dunnett T3 method. Variables with $P$ values $<0.10$ were entered multivariate LMM analyses. In LMM analyses, square of time variable was introduced to achieve a better fitness according to BIC values. Due to high correlation among the CSF parameters, only one parameter with the lowest $P$ value (CSF leukocyte $\geq 20$ cells $/ \mu \mathrm{L}$ ) was entered LMM analyses.

To validate the model derived from the LMM analysis, the bootstrap method was used. The complete datasets were randomly resampled for 1000 times, and the final LMM model was refit. Fixed effect (FE) with standard errors for each variable was calculated for each iteration and their average across all samples was calculated. To evaluate the effect of delayed teratoma removal and timing of (T)-SIRT regimen, $\Delta$ CASE and $\Delta$ mRS scores at 6 months and 1 year were compared among the groups using waterfall plot analysis and ANOVA. 

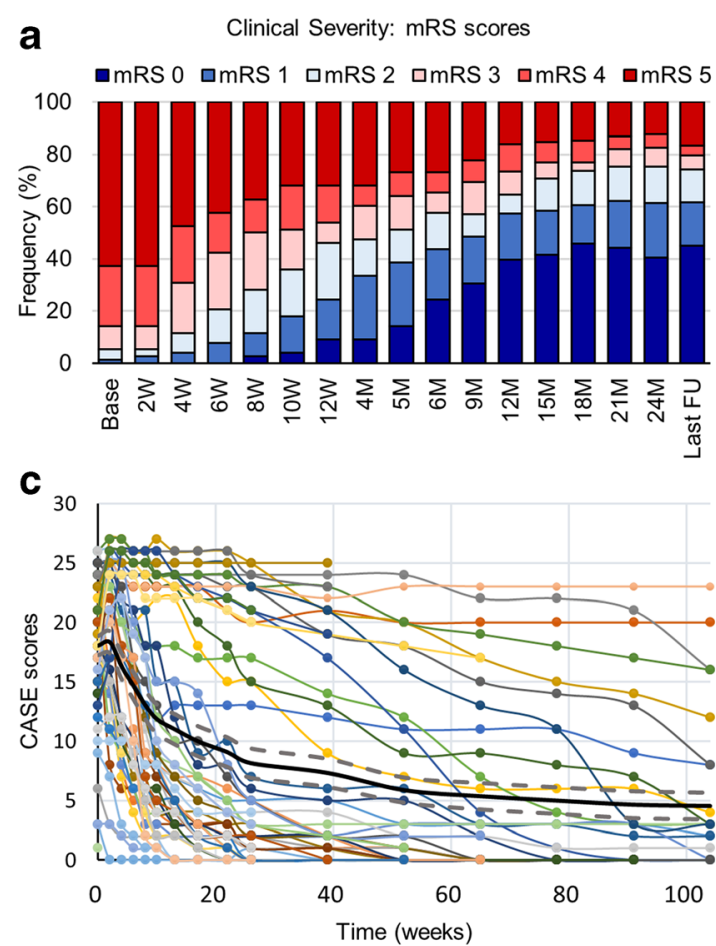

Fig. 1 Clinical courses and outcomes of the study population. Panel A demonstrates Clinical Assessment Scale in Autoimmune Encephalitis (CASE) score profiles and panel B demonstrates modified Rankin Scale (mRS) score profiles during the follow-up period. Panel C demonstrates CASE score changes of each patient. Black line indicates the mean CASE score and dashed lines indicate its $95 \%$ confidence intervals. In panel D,

To further adjust for the differences in the baseline severity among the patients, ANOVA analysis for the $\Delta$ CASE and $\Delta$ $\mathrm{mRS}$ scores was repeated for the subgroup with baseline $\mathrm{mRS}$ score of 5. R, version 3.6.0 (R Programming) and SPSS 25.0 (IBM Corp., Armonk, NY) were used for the statistical analyses and a two-tailed $P$ value $<0.05$ was considered to indicate significance.

\section{Data Availability}

The datasets generated or analysed during the current study are available on request from any qualified investigator.

\section{Results}

\section{Patient Characteristics}

Seventy-eight patients (60 [76.9\%] female and 18 [23.1\%] male, mean age of $27.4 \pm 13.4$ [range 6-78] years old) treated for anti-NMDAR encephalitis were identified. Every patient was hospitalized in SNUH during the acute period and followed up for more than 6 months. Seven hundred forty-two/780 (95.1\%) of the initial 6 months of data were obtained, and the rate of data acquisition for subsequent periods varied
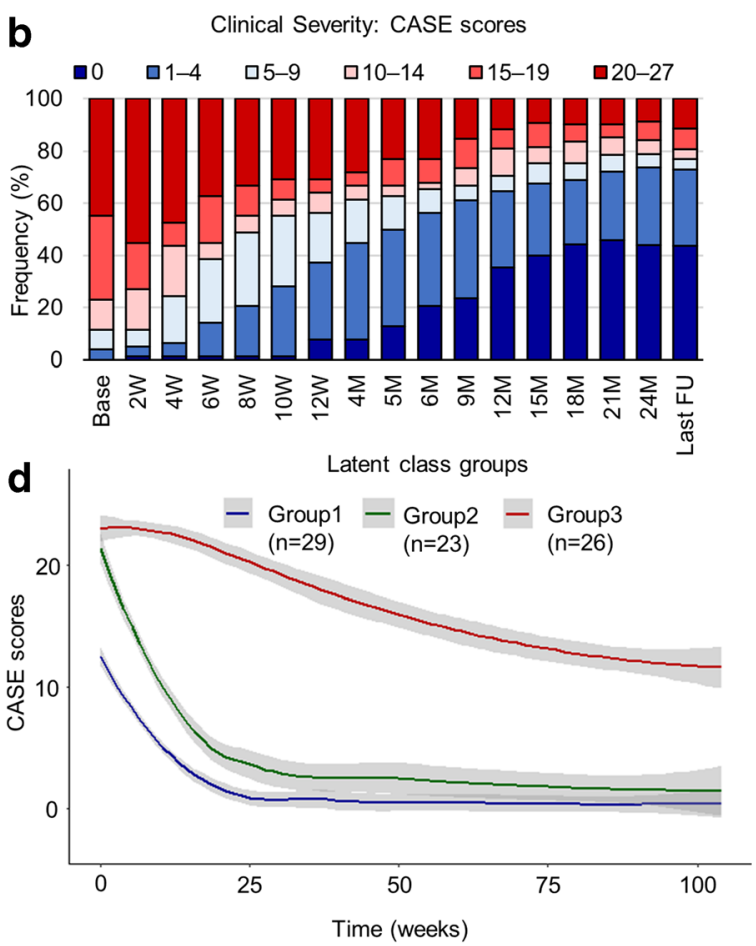

latent class linear modelling classified the patients into three groups according to the CASE score change patterns (group 1 [low initial severity + rapid improvement, $N=29$ ], group 2 [high initial severity + rapid improvement, $N=23$ ] and group 3 [high initial severity + delayed and insufficient improvement, $N=26]$ ). The gray-filled areas indicate the $95 \%$ confidence interval of the trend lines

according to the follow-up duration. At baseline, the median CASE score was 18.5 [15-23], and the median mRS score was $5[4,5]$. Twenty-six $(33.3 \%)$ patients exhibited refractory status epilepticus, and thirty-seven $(47.4 \%)$ received ventilator care. Thirty-one $(39.7 \%$, women $100.0 \%$ ) had teratomas (twenty-one unilateral ovarian teratomas, one bilateral ovarian teratoma and one mediastinal teratoma) and thirty $(96.8 \%)$ underwent surgical resection. No tumour other than a teratoma was identified.

First-line immunotherapy was initiated at 9.5 (5-22.3) days from the onset of symptoms. One patient has been stably treated for intermittent seizure and aggressive behaviour for 1 year and later developed the typical fullblown symptoms of anti-NMDAR encephalitis. In that case, the disease onset time was regarded according to that of the typical encephalitis symptoms (45 days), instead of the onset of the initial seizures (415 days). IVIG was administered in $75(96.2 \%)$ patients, steroids in $69(88.5 \%$, oral prednisolone maintenance in 20 [25.6\%] patients) for mean $4.8 \pm 0.6$ days of duration and plasmapheresis in 2 (2.6\%). Rituximab was administered in $72(92.3 \%)$ patients for a median of 6 [4-11] cycles at 12 (7-19.8) days from the initiation of first-line immunotherapy. Tocilizumab was administered in $52(66.7 \%)$ patients for a median of 4 [2-6] cycles at 36 (14-64) days from the 


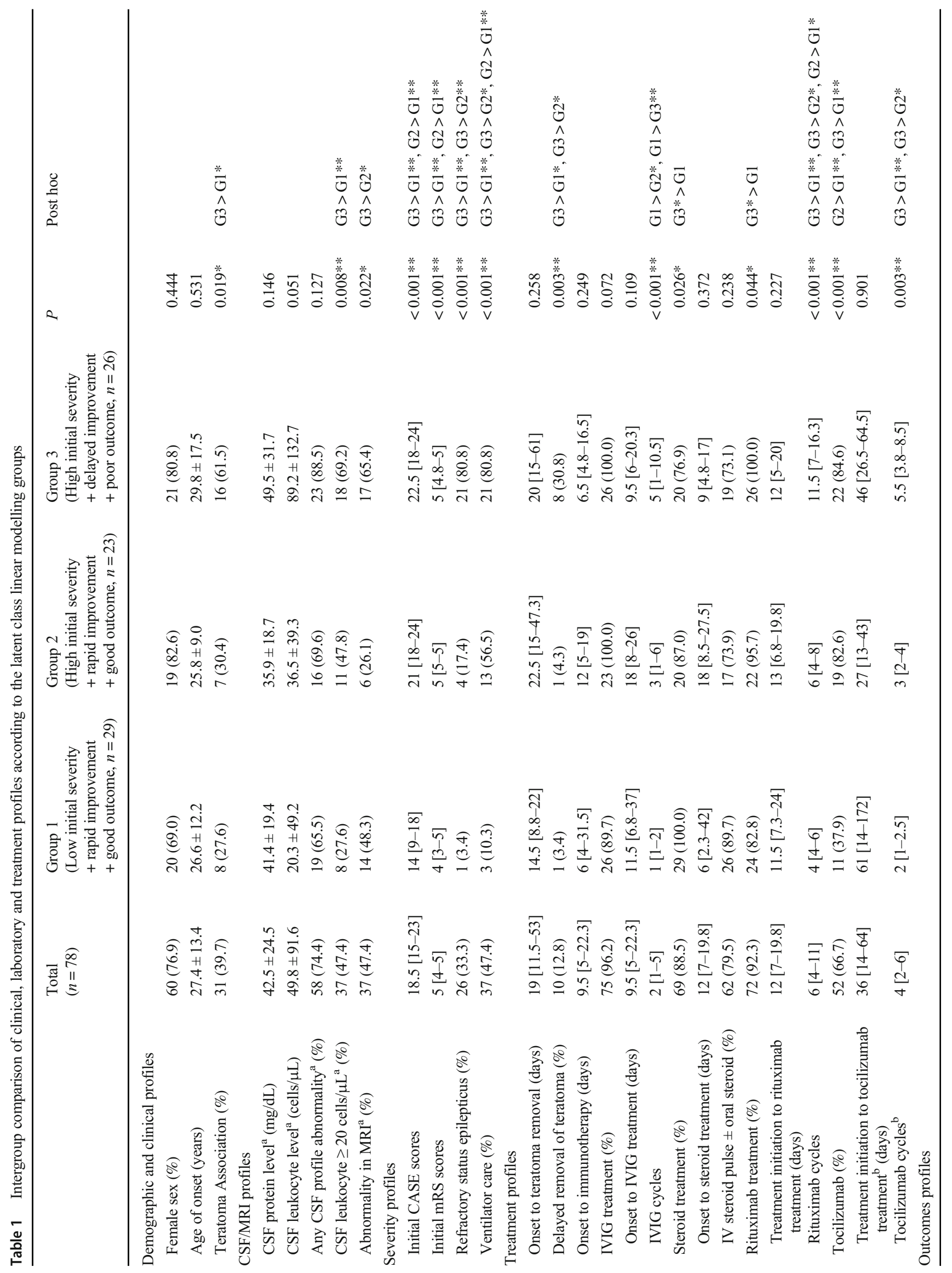




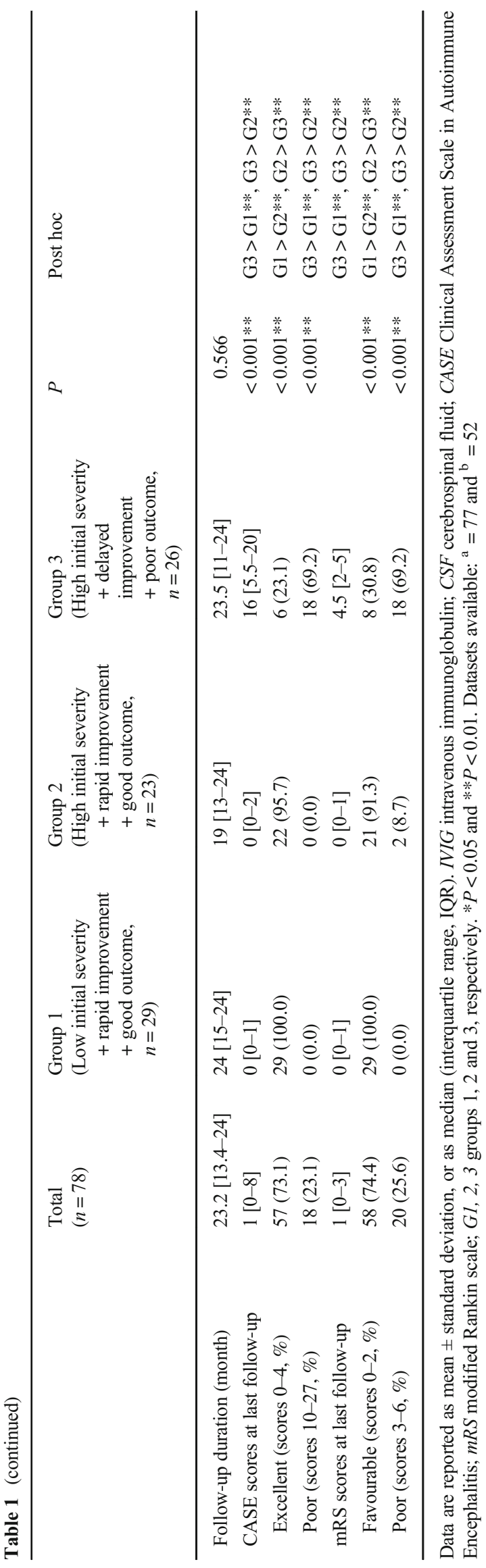

first-line immunotherapy initiation. The number of patients completed each regimen at each time point for the first 12 months are summarized in Supplemental Table 1.

The mean duration of follow-up was 23.2 (13.4-24) months (range 6-24 months). At the last follow-up, the median CASE score was $1(0-8)$, and the median mRS score was 1 (0-3). The frequency of excellent outcomes was $57(73.1 \%)$, while that of poor outcome was $18(23.1 \%)$ according to the CASE scores, and that of favourable outcomes was 58 (74.4\%), while that of poor outcomes was $20(25.6 \%)$ according to mRS scores (Fig. 1A, B). The observed clinical, laboratory, treatment and outcome profiles are summarized in Table 1.

\section{Factors Associated with CASE Score Changes Over Time}

When the changes in CASE scores were plotted over time, major improvement occurred during the first 6 months, although improvement after that time was relatively minor (Fig. 1C). LCLM analysis classified twenty-nine patients into group 1 (low initial severity + rapid improvement + good outcome), twenty-three patients into group 2 (high initial severity + rapid improvement + good outcome) and twenty-six patients into group 3 (high initial severity + delayed improvement + poor outcome, Fig. 1D). In the ANOVA analyses, group 3 was associated with a higher frequency of ovarian teratoma, abnormal findings in CSF or on MRI analyses, refractory status epilepticus, ventilator care and delayed removal of teratoma. Although group 3 received more cycles of IVIG, rituximab and tocilizumab, the outcome was the worst (Table 1).

In multivariate LMM analysis including the factors associated with poor outcomes in Table 1, time was inversely correlated with CASE scores (FE - 0.205 score/week, 95\% confidence interval $[\mathrm{CI}]-0.241$ to -0.169 score/week, $P<0.001$ ), indicating the monophasic features of the disease. When the completed immunotherapy regimens were counted at each outcome time point, more combinations were associated with lower CASE scores (FE - 1.684, 95\% CI - 2.365 to $-1.003, P<0.001$ for SIRT vs. SI; FE $-1.009,95 \%$ CI 1.596 to $-0.423, P=0.001$ for SIRT vs. SIR; and FE -0.675 , $95 \% \mathrm{CI}-1.161$ to $-0.012, P=0.045$ for SIR vs. SI). Additionally, CASE score at the baseline (FE 0.452 per 1 score increment in the baseline CASE score, 95\% CI 0.237 $0.666, P=0.010$ ), the presence of refractory status epilepticus (FE 6.036, 95\% CI 3.366-8.705, $P<0.001$ ) and the presence of a teratoma (FE 1.349, 95\% CI 0.566-2.131, $P=0.001$ ) were associated with higher CASE scores at each time point. However, ventilator care, abnormal findings on MRI or CSF leukocytes $\geq 20$ cells $/ \mu \mathrm{L}$ were not significantly associated with CASE scores at each time point (Table 2). Bootstrap validation performed with 1000 iterations also reproduced the results (Supplemental Table 2). 
Table 2 Linear mixed models for the longitudinal CASE score changes

\begin{tabular}{llll}
\hline & $\begin{array}{l}\text { Coefficient for the fixed } \\
\text { effect (mean } \pm \text { SE) }\end{array}$ & $95 \%$ confidence interval & $P$ \\
Model $1^{\mathrm{a}}$ & & & Post hoc \\
\hline Intercept & $4.141 \pm 2.217$ & -0.268 to 8.549 & 0.065 \\
Age (years) & $0.016 \pm 0.042$ & -0.068 to 0.099 & 0.708 \\
Male sex & $0.110 \pm 1.269$ & -2.414 to 2.633 & 0.931 \\
Baseline CASE scores & $0.452 \pm 0.108$ & 0.237 to 0.666 & $<0.001^{* *}$ \\
Time (weeks) & $-0.205 \pm 0.018$ & -0.241 to -0.169 & $<0.001^{* *}$ \\
Time ${ }^{2}$ & $0.001 \pm 0.000$ & 0.001 to 0.001 & $<0.001^{* *}$ \\
Regimens (completed at each time point ${ }^{\ddagger}$ ) & & - \\
SI regimen & Reference & - & $0.010^{*}$ \\
SIR regimen & $-0.675 \pm 0.261$ & -2.365 to -1.003 & $<0.001^{* *}$ \\
SIRT regimen & $-1.684 \pm 0.347$ & 3.366 to 8.705 & $<0.001^{* *}$ \\
Refractory status epilepticus & $6.036 \pm 1.342$ & 0.566 to 2.131 & $0.001^{* *}$ \\
Presence of teratoma & $1.349 \pm 0.399$ & -2.138 to 2.417 & 0.903 \\
CSF leukocyte $\geq 20$ cells $/ \mu \mathrm{L}$ & $0.140 \pm 1.145$ & -0.701 to 4.431 & $0.001^{\dagger}$ \\
Ventilator care & $1.865 \pm 1.290$ & -1.471 to 2.977 & 0.152 \\
Abnormality in MRI & $0.753 \pm 1.118$ & & 0.503 \\
\hline
\end{tabular}

Bayesian information criterion (BIC) value of the models, a smaller value indicates a better fitness of the model: ${ }^{\text {a }}=5116.8$. SIRT steroid, intravenous immunoglobulin (IVIG), rituximab and tocilizumab; SIR steroid, IVIG and rituximab; SI steroid and IVIG; CSF cerebrospinal fluid; CASE Clinical Assessment Scale in Autoimmune Encephalitis. ${ }^{\star}$ Each evaluation time point was designated as follows: at the time of initiation of immunotherapy (baseline), every 2 weeks to 12 weeks, every month until the next 3 months and then every 3 months for the 12 months. $* P<0.05$, ** $P<0.01$ and ${ }^{\dagger}$ fixed effect (FE) $-1.009,95 \%$ confidence interval $(\mathrm{CI})-1.596$ to -0.423 for SIRT regimen versus SIR regimen

\section{Impact of Delayed Teratoma Removal}

Among the sixty female patients, the initial workup for teratoma was CT-only in 23 (38.3\%) patients, MRI-only in 12 (20.0\%), a combination of CT and MRI in $24(40.0 \%)$ and sonography in $1(1.7 \%)$. Thirteen teratomas were detected on initial CT (12 pelvis CT and one chest CT for mediastinal teratoma). Seven teratomas were detected by pelvis MRI and one by sonography. However, remaining nine (26.1\%) teratomas were not detected by initial pelvis $\mathrm{CT}$ (four, $44.4 \%$ ) or by combined pelvis CT and MRI (five, 55.6\%) but were detected in a follow-up pelvis MRI, and all of these cases had delayed removal of the teratoma (median time lag from onset to teratoma removal of 61 [53-206] days). One additional patient had teratoma on an initial pelvic MRI that was missed, and that patient did not receive teratoma removal. In those ten patients, despite rapid elevation of the treatment regimen to SIRT and removal of the teratoma at a later time,
Fig. 2 Change of CASE scores in patients with delayed teratoma removal. The arrow heads indicate the time points of teratoma removal. The line colours indicate the treatment regimen used at that time. $\mathrm{SI}=$ steroid and intravenous immunoglobulin (IVIG); SIR = steroid, IVIG and rituximab; and SIRT = steroid, IVIG, rituximab and tocilizumab

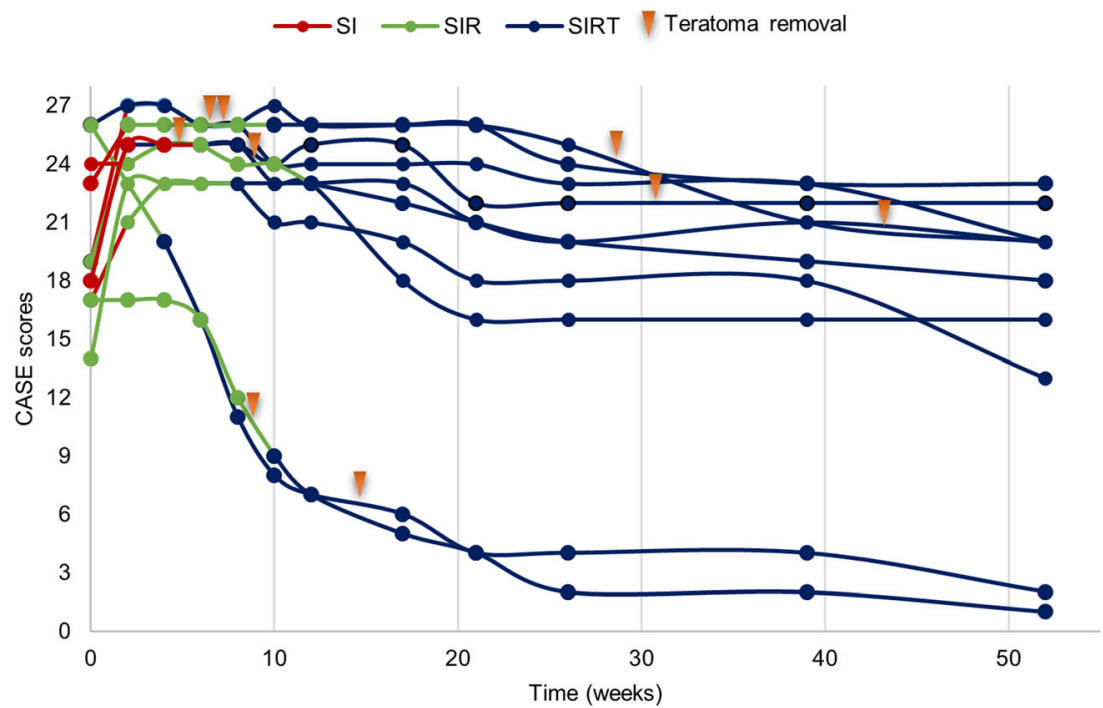




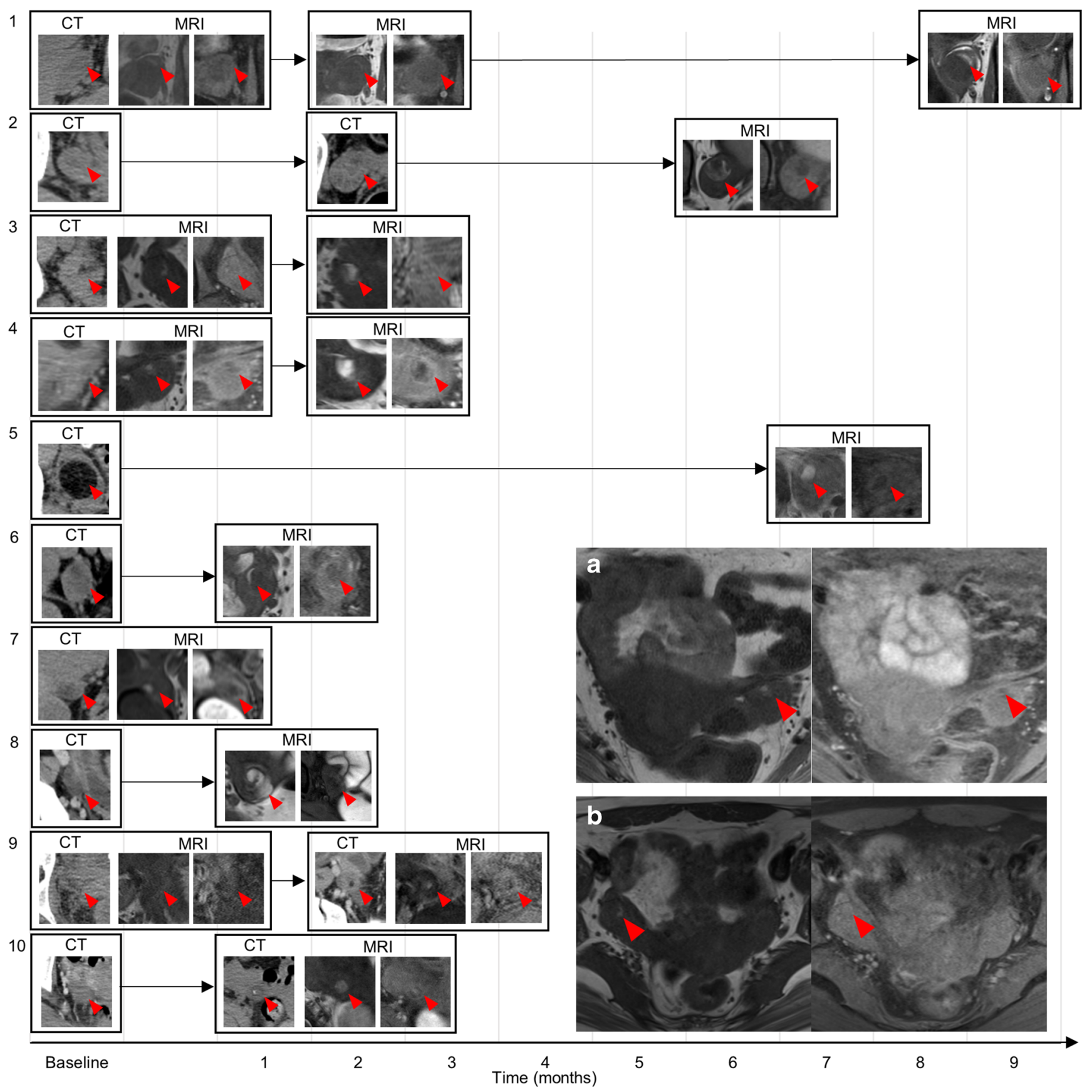

the clinical severity did not significantly improve over time except for in two patients (Fig. 2). In all cases, very small ovarian fat-containing tissues were present in the pelvic MRI T1 image (Fig. 3). The clinical profiles of the patients and the reasons for the delayed removal of teratoma are demonstrated in Fig. 3 and Table 3.

\section{Effect of Timing of (T)-SIRT Regimen}

To evaluate the effect of timing of the (T)-SIRT regimen, patients were categorized into (T)-SIRT, (T)-SIR/(T)-SI and SIRT/SIR with delayed teratoma removal according to the regimens completed after 1 month, 3 months or 1 year. When the (T)-SIRT regimen was completed in 1 month, the improvement in CASE score was the most prominent: the initially higher CASE scores reported for this group compared to the (T)-SIR/(T)-SI group were reversed over time (Fig. 4A). However, the completion of (T)-SIRT within 3 months or 1 year did not catch up to the initial score differences when this group was compared to the (T)-SIR/(T)-SI groups (Fig. 4B, C). In every categorization of regimens, delayed teratoma removal was associated with poor improvement of CASE scores over time (Fig. 4A-C). This trend was again noted in waterfall plot analyses showing individual changes of CASE 
Wig. 3 Imaging findings of the patients with delayed removal of teratoma. Every pelvis magnetic resonance image (PMRI) is provided in pairs, of which the left side indicates T1 weighted-image (T1) and the right side indicates fat-suppression $\mathrm{T} 1$ image. In right lower panels, subtle lesions with T1 high signal-intensity (HSI) are identified at left ovary (less than $4 \mathrm{~mm}$, red arrowheads, panel A) and at right ovary (5 mm, red arrowheads, panel B). The HSI was disappeared in the fat saturation images (right panels), which indicates a teratoma. In patient 1, no definite lowattenuation lesion at left ovary was identified in initial pelvis computed tomography (left, first images). Subtle T1 HSI with fat saturation at left ovary was suspected in initial PMRI (right, first images), which indicates a possible ovarian teratoma. However, the diagnosis was not made. In PMRI performed 2 months later, focal T1 HSI at left ovary was still subtle (second image). In PMRI performed 9 months later, T1 HSI with fat saturation at left ovary was identified (third image). In patient 2, no definite low-attenuation lesion at right ovary was identified in initial PCT (first image). There was still no significant finding in PCT performed 2 months later (second image). In PMRI performed 6 months later, T1 HSI with fat saturation at right ovary was identified (third image). In patient 3, no definite low-attenuation lesion at right ovary was identified in initial PCT and subtle T1 HSI with fat saturation at right ovary was suspected in initial PMRI (first images), but the diagnosis was not made. In PMRI performed 2 months later, T1 HSI with fat saturation at right ovary became very evident (second image). In patient 4 , no definite lowattenuation lesion at left ovary was identified in initial PCT (left, first image). Subtle T1 HSI with fat saturation at left ovary was suspected in initial PMRI (right, first image), but the diagnosis was not made. In PMRI performed 2 months later, T1 HSI with fat saturation at left ovary became very evident (second image). In patient 5 , low-attenuation lesion at right ovary with Hounsfield Unit (HU) - 199 was identified (first image) and the prompt right ovarian cystectomy under the diagnosis of ovarian teratoma was performed. However, 7 months later, follow-up PMRI revealed a new T1 HSI lesion with fat saturation at left ovary, which indicates a missed teratoma in the contralateral side (second image). In patient 6 , subtle low-attenuation lesion at left ovary was suspected in initial PCT (first image), but the diagnosis was not made. In PMRI performed 1 month later, T1 HSI with fat saturation at left ovary became evident (second image). In patient 7 , subtle low attenuation at left ovary with minimal HU -56 in initial PCT and also a small T1 HSI lesion with fat saturation at left ovary was suspected in initial PMRI (first images), but the diagnosis was not made. The image-based diagnosis of ovarian teratoma was retrospectively made at 5 years later, but the surgical removal of the teratoma was not performed (loss of follow-up). In patient 8 , subtle low attenuation at right ovary with minimal HU -62 in initial PCT was suspected (first images), but the diagnosis was not made. In PMRI performed 6 weeks later, T1 HSI with fat saturation at right ovary became evident (second image). In patient 9, no definite abnormality at right ovary was identified in initial PCT or in initial PMRI (first images). Follow-up PCT and PMRI performed 2 months later identified low attenuated T1 HSI lesion with fat saturation at right ovary (second image). In patient 10, no definite low-attenuation lesion at right ovary was identified in initial PCT (first images). Follow-up PCT and PMRI performed 6 weeks later identified mixed-attenuated T1 HSI lesion with fat saturation at right ovary (second image)

score at the 3-month, 6-month and 1-year outcome points (Supplemental Fig. 1).

In the ANOVA analyses, completion of (T)-SIRT within 1 month resulted in significantly higher $\triangle$ CASE scores $(P=0.012$ at 6 months and $P=0.015$ at 1 year) and $\Delta$ mRS scores (not significant at 6 months and $P=0.020$ at 1 year) than were achieved in the groups that received (T)-

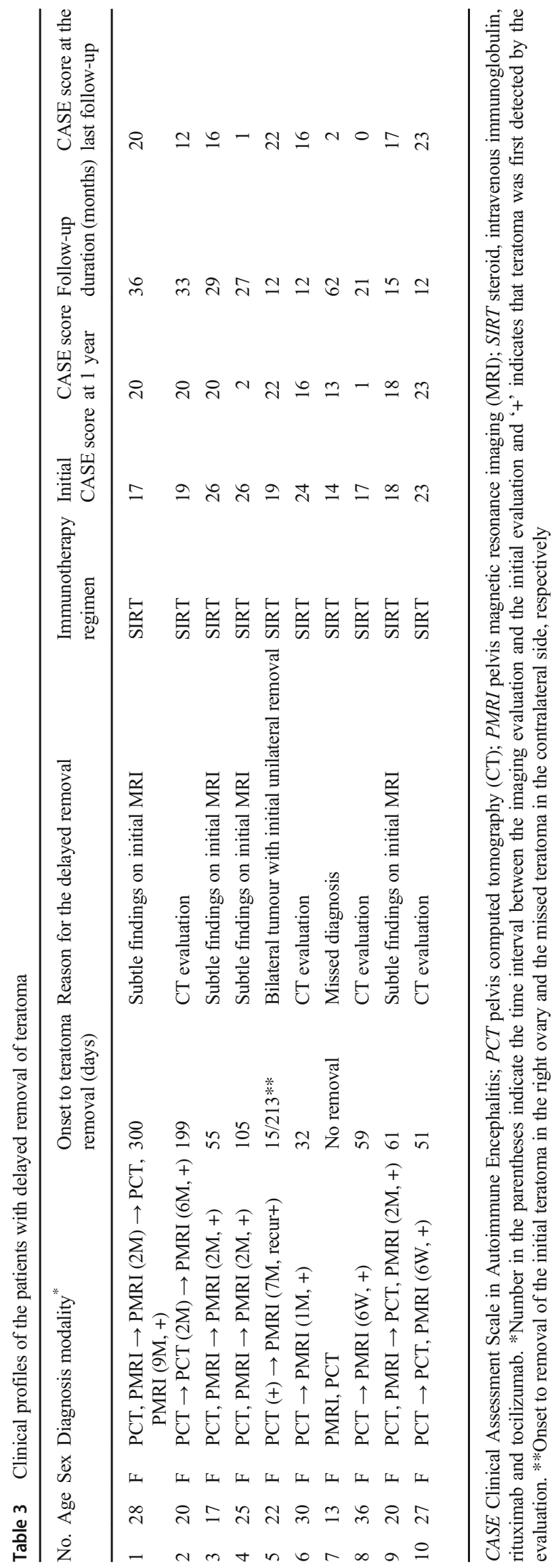



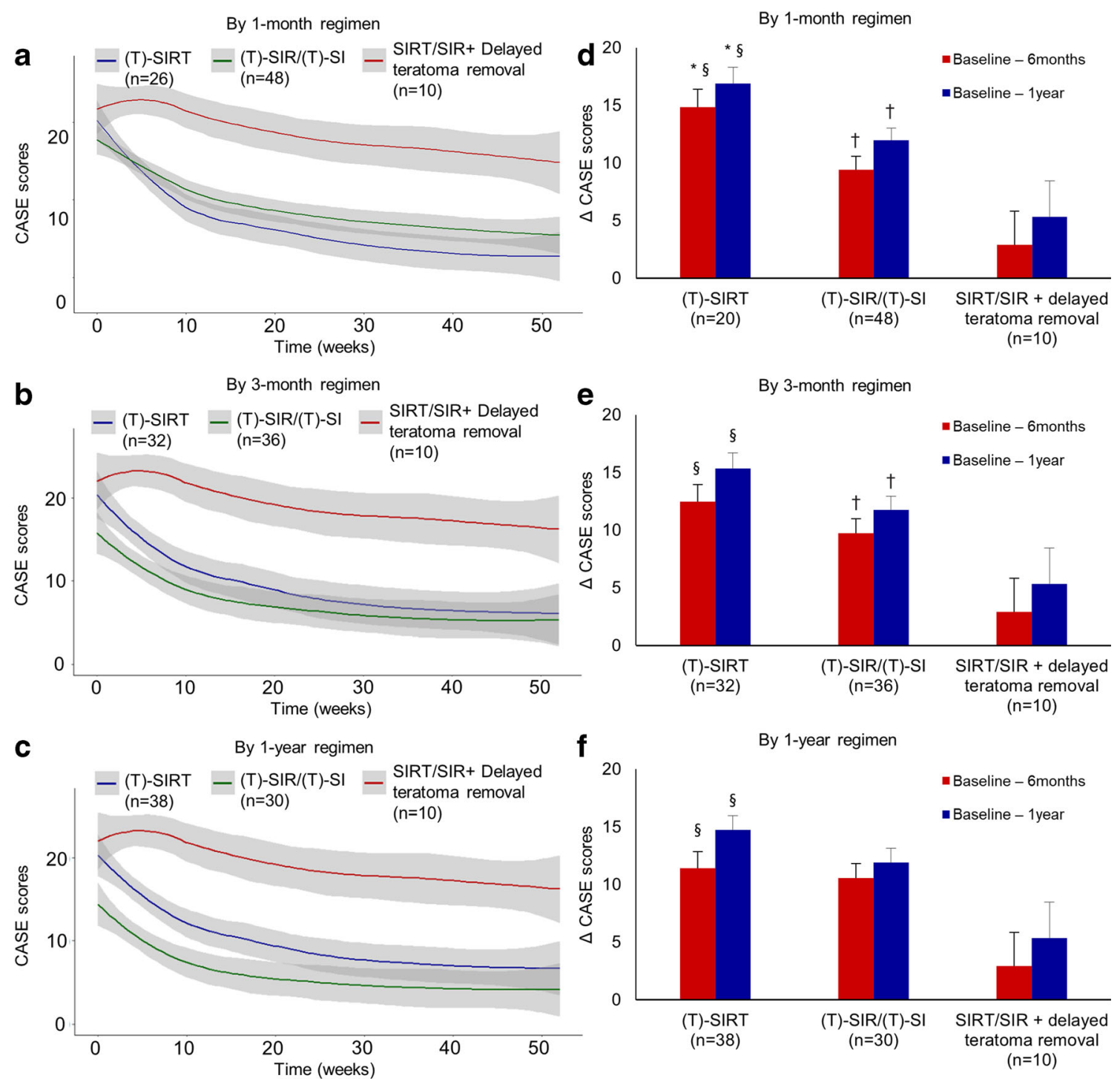

Fig. 4 Change of CASE scores for 1 year, according to the treatment regimens completed at 1 month, 3 months or 1 year. When (T)-SIRT regimen was completed in 1 month (blue line), initially higher CASE score compared to the (T)-SIR/(T)-SI group (green line) drops rapidly and the reversal crossing between the groups occurs over time (panel A). However, when the completion of (T)-SIRT was categorized as within 3 months or 1 year (panels B and C), they failed to catch up the initial score differences against (T)-SIR/(T)-SI groups. In every category of regimens, delayed teratoma removal (red lines) was associated with slower improvement of CASE scores over time. The gray-filled areas

indicate the $95 \%$ confidence interval of the trend lines. In panels D, E and $\mathrm{F}$, ANOVA analyses for the 6-month $\Delta$ CASE scores and 1-year $\Delta$ CASE scores among the groups categorized according to the regimens completed at 1 month, 3 months or 1 year were demonstrated. $(\mathrm{T})=$ teratoma removal; SI = steroid and IVIG; SIR = steroid, IVIG and rituximab; and SIRT $=$ steroid, IVIG, rituximab and tocilizumab. $* P<0.05$ for (T)-SIRT versus (T)-SIR/(T)-SI, ${ }^{\dagger} P<0.05$ for (T)-SIR/(T)-SI versus SIRT/SIR + delayed teratoma removal and ${ }^{\S} P<0.05$ for SIRT/SIR + delayed teratoma removal versus (T)-SIRT

SIR/(T)-SI within 1 month (Supplemental Table 3). However, the delayed completion of (T)-SIRT in 3 months or 1 year had no significant impact on the 6-month or 1year outcomes with regard for CASE scores. In every analysis, delayed teratoma removal was uniformly associated with the smallest 6-month and 1-year changes in CASE scores (Fig. 4D-F). The patients' clinical, laboratory and treatment profiles (except for tocilizumab treatment) were comparable among the groups separated per the 1-month treatment regimen (Supplemental Table 3).
In the subgroup analysis for the patients with baseline mRS score of 5, completion of (T)-SIRT within 1 month again showed significantly higher $\Delta$ CASE scores and $\Delta \mathrm{mRS}$ scores at 1 year than were achieved in the groups that received (T)-SIR/(T)-SI within 1 month $(P=0.032$ and $P=0.019$, respectively), although the 6-month $\Delta$ score differences were not significant (Fig. 5). The baseline risk factor profiles were comparable between the two groups (Supplemental Table 4). In LMM analysis, more combinations of immunotherapy were again associated with lower CASE scores, while 


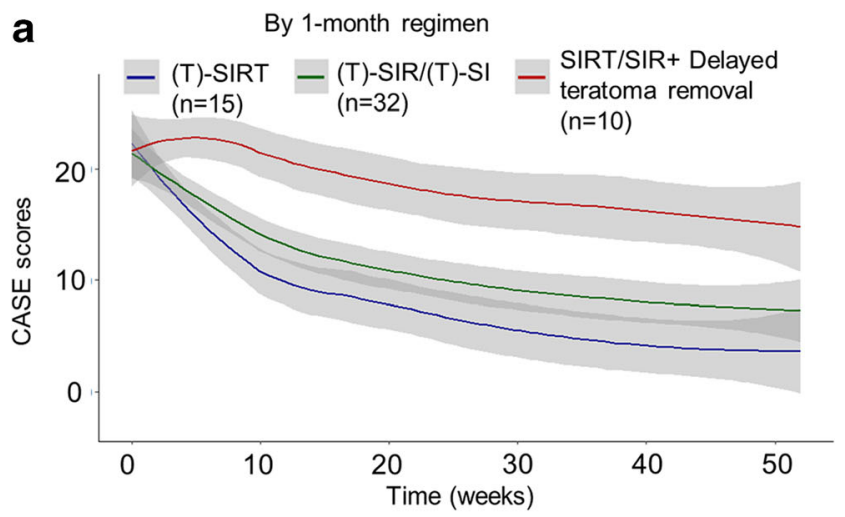

b By 1-month regimen

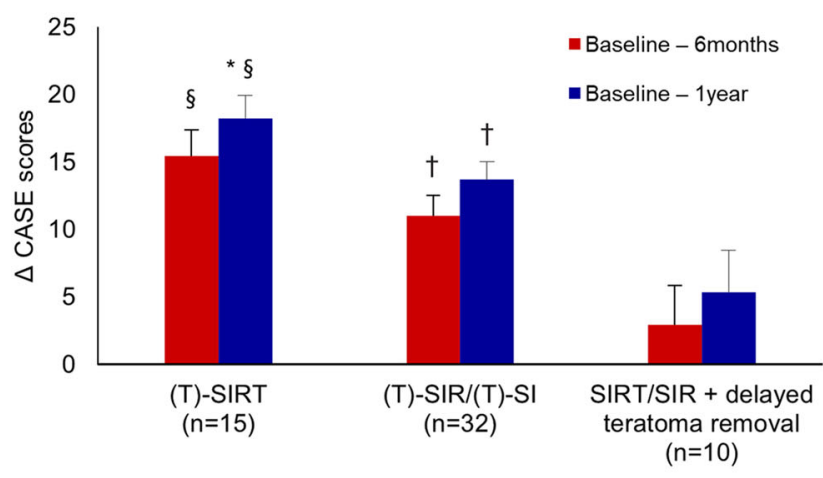

C

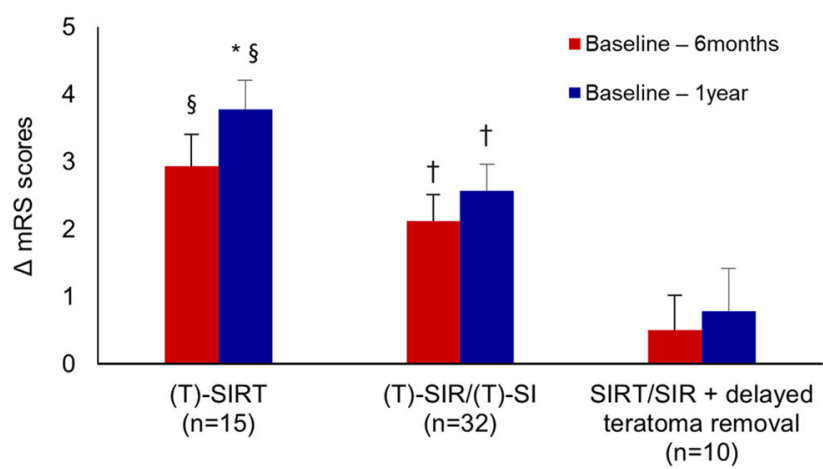

Fig. 5 Change of CASE scores and mRSS scores according to the treatment regimens completed at 1 month in the subgroup with baseline mRS score of 5. In panel A, (T)-SIRT regimen completed in 1 month (blue line) showed higher rate of CASE score improvement compared to the (T)-SIR/(T)-SI group (green line) or the delayed teratoma removal (red lines). The gray-filled areas indicate the $95 \%$ confidence interval of the trend lines. ANOVA analyses show that 1-year $\triangle$ CASE scores (panel B) and $\Delta \mathrm{mRS}$ scores (panel C) were higher in the group that used (T)SIRT regimen within 1 month compared to the other treatment status. (T) = teratoma removal; SI = steroid and IVIG; SIR = steroid, IVIG and rituximab; and SIRT = steroid, IVIG, rituximab and tocilizumab. $* P<0.05$ for (T)-SIRT versus (T)-SIR/(T)-SI, ${ }^{\dagger} P<0.05$ for $(\mathrm{T})-\mathrm{SIR} /$ (T)-SI versus SIRT/SIR + delayed teratoma removal and ${ }^{\S} P<0.05$ for SIRT/SIR + delayed teratoma removal versus (T)-SIRT

refractory status epilepticus and the presence of a teratoma were also significantly associated with higher CASE scores (Supplemental Table 5).

\section{Safety Profiles}

Ninety-nine adverse events developed during follow-up. Pneumonia was the most common, followed by urinary tract infection, neutropenia, lymphopenia, acute kidney injury and eosinophilia. Pneumonia was relatively more common during the early treatment phases (prior to immunotherapy or during SI), whereas neutropenia, lymphopenia and urinary tract infection were common in the later phases (during SIR or SIRT). Serious adverse events developed in one patient (decrease in the neutrophil count) during SIRT regimen and tocilizumab was withdrawn after the fourth administration cycle three of them (Supplemental Table 6).

\section{Discussion}

This study suggests several therapeutic points to improve the outcomes in anti-NMDAR encephalitis. The SIRT regimen, including tocilizumab in addition to steroids, immunoglobulin and rituximab, was more effective than was observed for conventional regimens without tocilizumab, such as SIR or SI, and the fast completion of SIRT within 1 month provided the best results. Delayed application of the combination immunotherapy was less effective. In addition, delayed removal of teratoma for more than 1 month was associated with poor outcomes. Overall, the completion of the (T)-SIRT regimen within 1 month was effective in achieving better 6-month and 1 -year outcomes, according to the changes in both $\mathrm{mRS}$ and CASE scores. Although a decreased neutrophil count during the SIRT regimen and a decreased lymphocyte count during the SIR regimen were adverse effects observed in this study, the combination immunotherapy was well tolerated.

The efficacy of the combination of SIRT might be explained by the different mechanisms of each regimen, which can compensate for each other. Steroids suppress activated lymphocytes, thus reducing pro-inflammatory cytokines and effector cell differentiation [21, 24]. IVIG enhances the clearance of pathogenic IgG by saturating the neonatal Fc receptor, which internalizes IgG and rescues it from lysosomal degradation [25-27]. IVIG also blocks Fc gamma receptors (Fc $\gamma \mathrm{R})$ via saturation, upregulates type IIB Fc $\gamma \mathrm{R}$ which inhibits antibody generation by B cells, neutralizes autoantibodies, downregulates the cytokine network and interferes with $\mathrm{T}$ and B cell activation [26, 27]. Rituximab depletes CD20positive activated and memory B cells, thus blocking the germinal centre reaction in anti-NMDAR encephalitis [7, 21, 28]. In a recent meta-analysis for the treatment of AE or neuromyelitis optica, rituximab was established as an effective secondline agent with a favourable safety [29, 30]. However, rituximab cannot target CD20-negative activated plasma cells [8, 31 , which could prolong the disease duration even when firstline and second-line immunotherapies are applied. 
Tocilizumab binds to both soluble and membrane-bound IL-6 receptors [32] and has multiple immunotherapeutic actions, such as the inhibition of $B$ cell activation and cytotoxic T cell differentiation, and IL-17-producing Th17 cell proliferation as well as the promotion of regulatory $\mathrm{T}$ cell differentiation $[8,9$, 21, 32-35]. In particular, plasma cells need IL-6 for survival, and tocilizumab can deplete IL-6-mediated survival in plasma cells [34]. Therefore, the simultaneous use of the SIRT regimen might maximize the coverage of the diverse mechanisms of $\mathrm{AE}$, enable a synergistic interaction among each regimen and thereby comprehensively restrain activated autoimmune cascades, and provide the highest chance of converting the patient to a quiescent status, especially in acute stages.

Previous studies have demonstrated the rescuing effects of rituximab or tocilizumab in patients unresponsive to first- or second-line immunotherapies, although the definition of unresponsiveness was based on mRS scores or the attending physician's decision without clear criteria [3, 6-8]. However, mRS scores might not be suitable for measuring clinical responsiveness to immunotherapy because they do not estimate the complex spectrum of symptoms observed in $\mathrm{AE}$ and have a ceiling effect $[3,11,36]$. Previous studies have also set the timing of determining unresponsiveness to a firstline immunotherapy at 4 weeks from initiation and to rituximab as 1 month from the last weekly session $[3,7,8]$. However, in critically ill patients, suspending the next-line immunotherapy for several weeks until they are confirmed to be unresponsive to the current immunotherapy might be unrealistic in acute stages. In this regard, the major advantage of the current study is that it is the first to demonstrate the effectiveness of a combination immunotherapy without trying to apply criteria for the stepwise elevation of the regimen.

As different immunotherapies are used for short intervals, the traditional approach to separately evaluate the effect of each immunotherapy might be inappropriate. To overcome this issue, we constructed a detailed time course of the disease, used CASE scoring system that is more precise and quantitative than $\mathrm{mRS}$ and performed LMM analysis which enables comparing the effect of each combination immunotherapy regimen on CASE score changes over time. Despite those advantages of LMM analysis, there are several issues that require careful interpretation of the result. Difference in the baseline severity, naturally recovering course of disease, nonrandomized choice of immunotherapy regimens and potential floor effect of CASE score system might have been potential sources of bias. ANOVA analyses for $\Delta$ CASE and $\Delta \mathrm{mRS}$ scores were performed to address those issues, by comparing the treatment status at a given time point (1 month), evaluating the outcomes at fixed time points ( 6 months and 1 year) and using both mRS and CASE systems. The subgroup analysis for the baseline mRS score of 5 was performed to adjust the heterogeneous baseline severity. However, effect of completing T-SIRT at later ( $>1$ month) times might have been underestimated by fixing the endpoint as 6 months and 1 year. For that, we compared the $\Delta$ CASE score among the 3-month treatment regimens, but completing (T)-SIRT within 3 months was not associated with increased $\triangle$ CASE scores during the remaining 3 month (by 6-month $\triangle$ CASE score) or 9 months (by 1-year $\Delta$ CASE score), compared to using (T)-SIR/(T)-SI regimens. Additionally, the relatively small number of patients and the risk of overfitting in LMM results were at least partially resolved by bootstrap validation.

Delayed removal of a teratoma had a substantial impact on clinical outcomes. Ovarian teratoma can produce NR1-IgG and activates germinal centre reaction [28]. Therefore, if the removal of teratoma is delayed, the continuing antigen presentation induces the affinity maturation of the antibody, and the generation of long-lived plasma cells infiltrating into both bone marrow and brain, which make the patients nonresponsive to the later removal of teratoma and refractory to immunotherapy. To facilitate the prompt detection of teratomas, MRI might have advantages in detecting small-sized teratomas that can be missed by CT evaluations [20]. Given that T1-weighted fat saturation images along with standard T1 images have the highest sensitivity (92\%) in detecting ovarian teratoma [37], careful detection of ovarian fat-containing tissues using both standard and fat saturation T1 images is important. Additionally, as a small teratoma can be undetected in the initial evaluation, pelvic MRI should be repeated if the response to immunotherapy is suboptimal, although the initial workup for a teratoma returned a negative result. Furthermore, follow-up evaluations should be considered even after the teratoma has been removed to check for an occurrence of disease in the contralateral side, as was found in the case of one patient (patient 5) in this study.

The presence of refractory status epilepticus was associated with 6.0-point higher CASE scores. Considering the epileptogenic properties of anti-NMDAR antibodies and that refractory status epilepticus induces permanent damage in neurons and synapses via mechanisms distinct from those involved in $\mathrm{AE}[9,13,38]$, early completion of SIRT to control seizures with continuous monitoring might be important to achieve good outcomes.

Adverse events were frequent during the disease course. The events occurring at the early treatment phases might be related with the disease severity, which includes decreased consciousness, brainstem dysfunction and seizure-related complications. Development of neutropenia or lymphopenia in the later treatment phases might be highly associated with the repetitive use of combination immunotherapy and therefore warrants regular surveillance and management. The frequency of neutropenia during the SIRT regimen was higher than that of the previous study observed during tocilizumab treatment $(11 / 52,21.2 \%$ vs. $3 / 30,10.0 \%)$ [8], which might be partly attributable to the concomitant use of rituximab, for which cycles ranged from 4 to 16 at the time that the 
leukopenia developed. However, SIRT was well tolerated in all but one patient, and no life-threatening events were observed. Because IL- 6 mediates acute inflammatory reaction and stimulates the production of inflammatory cytokines [35], signs of infection might be masked when using tocilizumab. Close follow-up with surveillance for infectious complications is therefore necessary during SIRT treatment.

To further improve and validate our findings, a prospective randomized controlled study might be necessary. However, the findings from cohort studies, such as the current one, might be a good evidence for treatment strategies to date. Additionally, more immune therapy options such as proteasome inhibitors (such as bortezomib), anti-CD19 agents (such as inebilizumab) or novel IL-6 receptor blocker (satralizumab) could be added to T-SIRT regimen [21,39-43].

Required Author Forms Disclosure forms provided by the authors are available with the online version of this article.

Funding This work was supported by a National Research Foundation of Korea (NRF) grant funded by the Ministry of Science, ICT \& Future Planning, Republic of Korea (2019R1A2C1009003).

\section{Compliance with Ethical Standards}

Competing Interests The authors declare that they have no competing interests.

\section{References}

1. Dalmau J, Graus F. Antibody-mediated encephalitis. New England Journal of Medicine 2018;378:840-851.

2. Dalmau J, Gleichman AJ, Hughes EG, et al. Anti-NMDA-receptor encephalitis: case series and analysis of the effects of antibodies. The Lancet Neurology 2008;7:1091-1098.

3. Titulaer MJ, McCracken L, Gabilondo I, et al. Treatment and prognostic factors for long-term outcome in patients with anti-NMDA receptor encephalitis: an observational cohort study. The Lancet Neurology 2013;12:157-165.

4. Irani SR, Bera K, Waters P, et al. N-methyl-D-aspartate antibody encephalitis: temporal progression of clinical and paraclinical observations in a predominantly non-paraneoplastic disorder of both sexes. Brain 2010;133:1655-1667.

5. Dubey D, Pittock SJ, Kelly CR, et al. Autoimmune encephalitis epidemiology and a comparison to infectious encephalitis. Annals of neurology 2018;83:166-177.

6. Dale RC, Brilot F, Duffy LV, et al. Utility and safety of rituximab in pediatric autoimmune and inflammatory CNS disease. Neurology 2014;83:142-150.

7. Lee W-J, Lee S-T, Byun J-I, et al. Rituximab treatment for autoimmune limbic encephalitis in an institutional cohort. Neurology 2016 https://doi.org/10.1212/WNL.0000000000002635.

8. Lee W-J, Lee S-T, Moon J, et al. Tocilizumab in autoimmune encephalitis refractory to rituximab: an institutional cohort study. Neurotherapeutics 2016;13:824-832.

9. Jun JS, Lee ST, Kim R, Chu K, Lee SK. Tocilizumab treatment for new onset refractory status epilepticus. Annals of neurology 2018;84:940-945.
10. Balu R, McCracken L, Lancaster E, Graus F, Dalmau J, Titulaer M. A score that predicts 1-year functional status in patients with antiNMDA receptor encephalitis. Neurology 2019;92:e244-e252.

11. Lim JA, Lee ST, Moon J, et al. Development of the clinical assessment scale in autoimmune encephalitis. Annals of neurology 2019;85:352-358.

12. Dalmau J, Armangué $\mathrm{T}$, Planagumà $\mathrm{J}$, et al. An update on antiNMDA receptor encephalitis for neurologists and psychiatrists: mechanisms and models. The Lancet Neurology 2019;18(11): 1045-1057

13. Gaspard N, Foreman BP, Alvarez V, et al. New-onset refractory status epilepticus: etiology, clinical features, and outcome. Neurology 2015;85:1604-1613.

14. Tuzun E, Zhou L, Baehring JM, Bannykh S, Rosenfeld MR, Dalmau J. Evidence for antibody-mediated pathogenesis in antiNMDAR encephalitis associated with ovarian teratoma. Acta Neuropathol 2009;118:737-743.

15. Graus F, Titulaer MJ, Balu R, et al. A clinical approach to diagnosis of autoimmune encephalitis. The Lancet Neurology 2016;15:391404.

16. Lee SK, Lee ST. The Laboratory Diagnosis of Autoimmune Encephalitis. J Epilepsy Res 2016;6:45-50.

17. Kim T-J, Lee S-T, Shin J-W, et al. Clinical manifestations and outcomes of the treatment of patients with GABA B encephalitis. Journal of neuroimmunology 2014;270:45-50.

18. Sunwoo J-S, Lee S-T, Byun J-I, et al. Clinical manifestations of patients with CASPR2 antibodies. Journal of neuroimmunology 2015;281:17-22.

19. Kim TJ, Lee ST, Moon J, et al. Anti-LGI1 encephalitis is associated with unique HLA subtypes. Annals of neurology 2017;81:183-192.

20. Saba L, Guerriero S, Sulcis R, Virgilio B, Melis G, Mallarini G. Mature and immature ovarian teratomas: CT, US and MR imaging characteristics. European journal of radiology 2009;72:454-463.

21. Shin Y-W, Lee S-T, Park K-I, et al. Treatment strategies for autoimmune encephalitis. Therapeutic advances in neurological disorders 2018;11:1756285617722347.

22. Common Terminology Criteria for Adverse Events (CTCAE v5.0). Available at: http://evsncinihgov/ftp1/CTCAE/ CTCAE 403 2010-06-14 QuickReference 5x7pdf;Accessed November 27, 2017.

23. Proust-Lima C, Philipps V, Liquet B. Estimation of extended mixed models using latent classes and latent processes: the $\mathrm{R}$ package lcmm. arXiv preprint arXiv:00890 2015.

24. Barnes PJ. How corticosteroids control inflammation: quintiles prize lecture 2005. British journal of pharmacology 2006;148: 245-254.

25. Jang Y, Lee S-T, Kim T-J, et al. High albumin level is a predictor of favorable response to immunotherapy in autoimmune encephalitis. Scientific Reports 2018;8:1012.

26. Schwab I, Nimmerjahn F. Intravenous immunoglobulin therapy: how does IgG modulate the immune system? Nature Reviews Immunology 2013;13:176.

27. Gelfand EW. Intravenous immune globulin in autoimmune and inflammatory diseases. New England Journal of Medicine 2012;367:2015-2025.

28. Makuch M, Wilson R, Al-Diwani A, et al. N-methyl-D-aspartate receptor antibody production from germinal center reactions: therapeutic implications. Annals of neurology 2018;83:553-561.

29. Nepal G, Shing YK, Yadav JK, et al. Efficacy and safety of rituximab in autoimmune encephalitis: a meta-analysis. Acta Neurol Scand 2020

30. Gao F, Chai B, Gu C, et al. Effectiveness of rituximab in neuromyelitis optica: a meta-analysis. BMC Neurology 2019;19:36.

31. Hachiya Y, Uruha A, Kasai-Yoshida E, et al. Rituximab ameliorates anti-N-methyl-D-aspartate receptor encephalitis by removal of 
short-lived plasmablasts. Journal of neuroimmunology 2013;265: 128-130.

32. Mihara M, Kasutani K, Okazaki M, et al. Tocilizumab inhibits signal transduction mediated by both mIL-6R and sIL-6R, but not by the receptors of other members of IL-6 cytokine family. International immunopharmacology 2005;5:1731-1740.

33. Cassese G, Arce S, Hauser AE, et al. Plasma cell survival is mediated by synergistic effects of cytokines and adhesion-dependent signals. The Journal of Immunology 2003;171:1684-1690.

34. Tanaka T, Kishimoto T. The biology and medical implications of interleukin-6. Cancer Immunology Research 2014;2:288-294.

35. Hunter CA, Jones SA. IL-6 as a keystone cytokine in health and disease. Nature Immunology 2015;16:448.

36. Finke C, Kopp UA, Prüss H, Dalmau J, Wandinger K-P, Ploner CJ. Cognitive deficits following anti-NMDA receptor encephalitis. J Neurol Neurosurg Psychiatry 2012;83:195-198.

37. Stevens S, Hricak H, Campos Z. Teratomas versus cystic hemorrhagic adnexal lesions: differentiation with proton-selective fat-saturation MR imaging. Radiology 1993;186:481-488.

38. Wright S, Hashemi K, Stasiak L, et al. Epileptogenic effects of NMDAR antibodies in a passive transfer mouse model. Brain 2015;138:3159-3167.
39. Behrendt V, Krogias C, Reinacher-Schick A, Gold R, Kleiter I. Bortezomib treatment for patients with anti-N-methyl-d-aspartate receptor encephalitis. JAMA neurology 2016;73:1251-1253.

40. Scheibe F, Prüss H, Mengel AM, et al. Bortezomib for treatment of therapy-refractory anti-NMDA receptor encephalitis. Neurology 2017;88:366-370.

41. Cree BA, Bennett JL, Kim HJ, et al. Inebilizumab for the treatment of neuromyelitis optica spectrum disorder (N-MOmentum): a double-blind, randomised placebo-controlled phase $2 / 3$ trial. The Lancet 2019;394:1352-1363.

42. Yamamura T, Kleiter I, Fujihara K, et al. Trial of satralizumab in neuromyelitis optica spectrum disorder. New England Journal of Medicine 2019;381:2114-2124.

43. Traboulsee A, Greenberg BM, Bennett JL, et al. Safety and efficacy of satralizumab monotherapy in neuromyelitis optica spectrum disorder: a randomised, double-blind, multicentre, placebo-controlled phase 3 trial. The Lancet Neurology 2020;19:402-412.

Publisher's Note Springer Nature remains neutral with regard to jurisdictional claims in published maps and institutional affiliations. 